\title{
Novel Enhanced-Thickness Magnetic Nanoparticle Thin-Films for System-On-Chip (SOC) Wireless Applications
}

\author{
Yuan $\mathrm{Li}^{1}$, Hyungie Doo ${ }^{2}$, Bo Pan ${ }^{1}$, Manos M. Tentzeris ${ }^{1}$, Z. John Zhang ${ }^{2}$, and John Papapolymerou ${ }^{1}$ \\ 1. Georgia Electronic Design Center, School of Electrical \& Computer Engineering, Georgia Institute of \\ Technology, Atlanta, GA 30332; \\ 2. School of Chemistry and Biochemistry, Georgia Institute of Technology, Atlanta, GA 30332
}

\begin{abstract}
In this paper, the propagation characteristics of an enhanced-thickness magnetic nanoparticle thin film are investigated on high resistivity silicon substrate $(10,000 \mathrm{ohm}-\mathrm{cm})$ for the first time up to $60 \mathrm{GHz}$. Contrary to other thin films, this nanoparticle thin film can achieve a thickness up to several hundred nanometers, even to micron. The enhanced thickness of this thin film is achieved by repeated coating of $\mathrm{CoFe}_{2} \mathrm{O}_{4}$ and poly, thus providing the thin film with the high permeability capable of potential practical wireless applications. Finite Ground Coplanar (FGC) waveguides are fabricated and characterized on silicon with the novel thin film. The measured attenuation and effective dielectric/magnetic constant are reported. Results show that the FGC waveguides with the thin film feature a lower attenuation compared to those on silicon for frequencies higher than $18 \mathrm{GHz}$. The extracted relative permeability has a value of 68 by matching of the effective dielectric/magnetic constant between simulation and measurement.
\end{abstract}

Index Terms - Magnetic thin film, Nanoparticle, Coplanar waveguide, high-permeability materials, FGC

\section{INTRODUCTION}

$\mathrm{D}$ riven by the demand of monolithic microwave integration, magnetic nanoparticle thin films [1]-[2] with high $\mu_{r}$ developed on CMOS compatible process, find numerous applications in high-speed wireless communication systems, (e.g. integrated inductors [3]-[5]), integrated electromagnetic noise countermeasures [6], and sensing [7]-[8].
In the traditional integrated spiral inductors with a good low quality factor, it is hard to achieve large inductance values even with an increased number of turns. As a result, a large area of the chip is occupied by inductors. Magnetic thin films, meanwhile, with high permeability, have the potential to provide compact integrated inductors with high inductance and good quality factors due to the reduced loss and the fact that the flux is constrained in the thin film instead of the whole substrate.

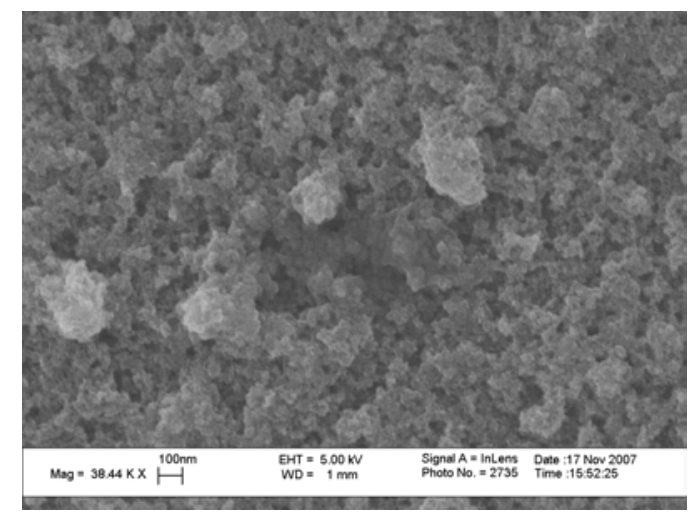

Fig. 1. The SEM picture of the enhanced-thickness magnetic nanoparticle thin film.

For integrated electromagnetic noise countermeasure applications, magnetic thin films provide a large attenuation in the desired stop band, while sustaining the insertion loss in the passband. For sensing applications, the large permeability of the thin film enhances its sensitivity.

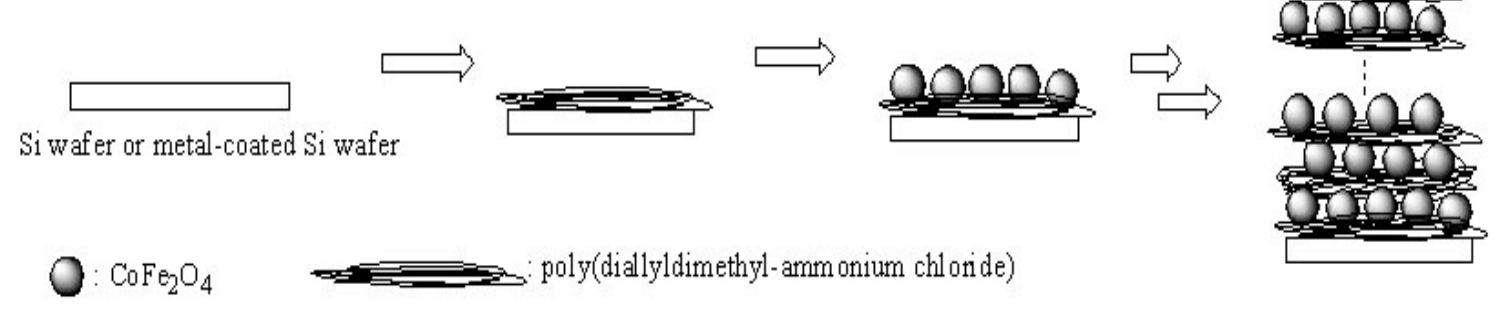

Fig. 2. Schematic of the thin film coating process. 
In this paper, an enhanced-thickness magnetic nanoparticle thin film is presented. This thin film is capable of allowing multiple nanoparticle layers up to several hundred nanometers, even up to a micron. The thin film presented here has 30 layers of nanoparticle with the size of $\sim 10 \mathrm{~nm}$ per nanoparticle. A smaller attenuation and larger extracted relative permeability of this thin film compared to those on silicon are observed in the experiment and simulation. This paper is organized as follows: the fabrication of the thin film and the CPW calibration lines are introduced in section II. The measurements of attenuation and effective dielectric/magnetic constant are discussed in section III. The simulation of the relative permeability of the thin film is presented in section IV, closing with the conclusions in section $\mathrm{V}$.

\section{Circuit FABRICATION}

The magnetic nanoparticle thin film was formed by taking the following four steps: The first step was the making of metal acetate $\left(\mathrm{Co}\left(\mathrm{C}_{2} \mathrm{H}_{3} \mathrm{O}_{2}\right)_{2}\right.$ \& $\left.\mathrm{Fe}\left(\mathrm{C}_{2} \mathrm{H}_{3} \mathrm{O}_{2}\right)_{3}\right)$. Metal salts $\left(\mathrm{CoCl}_{2}\right.$ $6 \mathrm{H}_{2} \mathrm{O} \& \mathrm{FeCl}_{3}$ ) were dissolved in $100 \mathrm{~mL}$ of distilled water and stirred until all salts dissolved. Then, $100 \mathrm{~mL}$ of $1 \mathrm{M} \mathrm{NaOH}$ was added into the solution and the solution was stirred for 1 hour. A precipitate was collected by centrifuging for 10 minutes and then washed with distilled water repeated for 4 times. The precipitate was dissolved in $25 \mathrm{~mL}$ of glacial acetic acid and then stirred until all dissolved. The solution was heated until it was dried. The solid $\left(\mathrm{Co}\left(\mathrm{C}_{2} \mathrm{H}_{3} \mathrm{O}_{2}\right)_{2} \& \mathrm{Fe}\left(\mathrm{C}_{2} \mathrm{H}_{3} \mathrm{O}_{2}\right)_{3}\right)$ was then collected and grounded.

The second step was the making of the nanoparticle, $\mathrm{CoFe}_{2} \mathrm{O}_{4}$. Metal acetates were added to $40 \mathrm{~mL}$ of dibenzyl ether and $10 \mathrm{~mL}$ of oleylamine and then stirred under $\mathrm{Ar}$ atmosphere at $140{ }^{\circ} \mathrm{C}$ for 1 hour to dissolve the metal acetates. The temperature of the solution was raised to $240{ }^{\circ} \mathrm{C}$ for 1 hour under Ar atmosphere. The solution was allowed to cool to room temperature. The particle was collected by a magnet, washed several times with an ethanol and then dried for 24 hours in air. The particle size was calculated from X-ray diffraction measurements and the quantitative chemical analysis was obtained by inductively coupled plasma measurements which showed the ratio of 1:2 between $\mathrm{Co}$ and $\mathrm{Fe}$.

Once the nanoparticle was formed, the third step was to prepare the two solutions for the making of the thin film. To remove the surfactants from nanoparticles, a solution containing nanoparticles $\left(\mathrm{CoFe}_{2} \mathrm{O}_{4}\right)$ in $\mathrm{pH} 12$ was stirred in the ultrasonicator (VCX 600, Sonics \& Matrials Inc.) for 1 hour. The nanoparticles were collected using a magnet was washed with a series of distilled water and ethanol. A solution was made with distilled water and collected nanoparticles. The polymer solution was diluted from poly.

The fourth step was the attachment of the thin film on the substrate. The thin film was formed on a silicon wafer with and without metal coating. The metal coated silicon wafer with thin film was for the capacitance measurements to determine the dielectric constant of the thin film and will be discussed later in this section. The thin film on the silicon wafer was used for measurements of the propagation characteristics, including attenuation and effective dielectric/magnetic constant.

The cleaned silicon wafer was dipped into the polymer solution for 5 minutes. Afterwards, the wafer was washed with a series of water and ethanol and then dried. The silicon wafer was put in the solution containing nanoparticle for 5 minutes. The unattached particles were removed with washes of water and ethanol, and the silicon wafer was dried. The coatings of the polymer and the nanoparticle for the thin film were alternatively repeated to get the desired thickness. The thickness of the film on the silicon wafer was measured in a profiler (KLA-Tencor P15). Fig. 1 gives the SEM picture of the thin film. Fig. 2 shows the schematic diagram of how to make thin film on the silicon wafer. The uniqueness of the process is the potential capability to build a film up to a few microns.

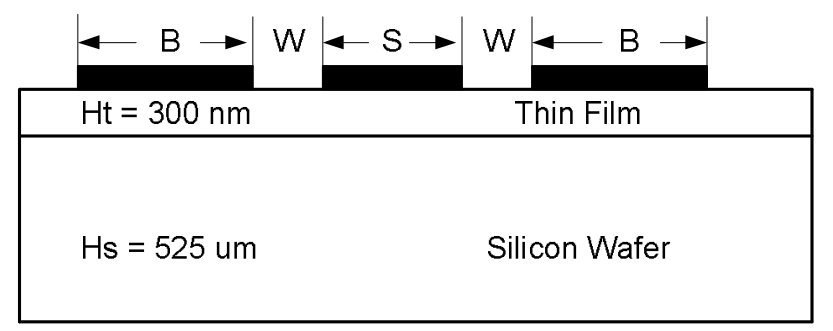

Fig. 3. Schematic of the finite coplanar waveguide on a high resistivity silicon wafer with nanoparticle thin film.

Once the nanoparticle thin film with the desired thickness was formed, the finite coplanar waveguide (FGC) was patterned on top of the thin film as a benchmarking topology. Different from [2], where the lift-off process was employed to obtain the finite coplanar waveguides, a silicon micromachined shadow mask was adopted to get the finite coplanar waveguide directly by using the E-Beam evaporator. The silicon shadow mask was used here to keep the thin film intact from the potential chemical contamination in lift-off process. The silicon shadow mask was fabricated on a low-resistivity silicon substrate. The finite coplanar waveguide pattern on the shadow mask was etched through using the DRIE process. The process to prepare the silicon shadow mask is the same as [9].

After the silicon shadow mask was obtained, it was mounted on the silicon wafer with thin film. The silicon wafer with the thin film was evaporated with $\mathrm{Ti} / \mathrm{Cu} / \mathrm{Au}$ for $20 \mathrm{~nm} / 1 \mathrm{um} / 20 \mathrm{~nm}$ using E-Beam evaporator. The schematic of the silicon wafer with thin film and metals are shown in Fig. 3. $S$ denotes the width of the signal line and is equal to $180 \mathrm{um}$, while $W$ denotes the width of the slot between the signal line and the ground, and is equal to 108 um. $B$ denotes the width of the ground and is equal to $500 \mathrm{um}$ for the benchmarking structures. 


\section{MEASUREMENTS}

The finite ground coplanar waveguide prototypes consisted of Thru-Reflect-Line (TRL) calibration standards with six delay lines of $2300 \mathrm{um}, 3200 \mathrm{um}, 4200 \mathrm{um}, 5300 \mathrm{um}, 6500 \mathrm{um}$, and 8000 um to cover the frequency range of $5-60 \mathrm{GHz}$. The NIST MULTICAL algorithm [10]-[11] was used in the calibration to compute the propagation constants, as

$$
\gamma=\alpha+j \beta
$$

where, $\gamma$ is the propagation constant, $\alpha$ is the attenuation constant, $\beta$ is the phase constant. The measurements were taken on an Agilent Vector Network Analyzer (VNA) 8510XF. A pair of GSG 250 um pitch probes was used in the measurement. The effective dielectric/magnetic constant including both effective permittivity and the effective permeability is extracted from MULTICAL. Although the relative permittivity and relative permeability of the thin film is unknown at this point, an increase of $\sim 10 \%$ in the effective dielectric/magnetic constant over a wide bandwidth is observed from the experiment in Fig. 4. Compared to [2], the effective dielectric/magnetic constant is increased in the hereby presented thin film.

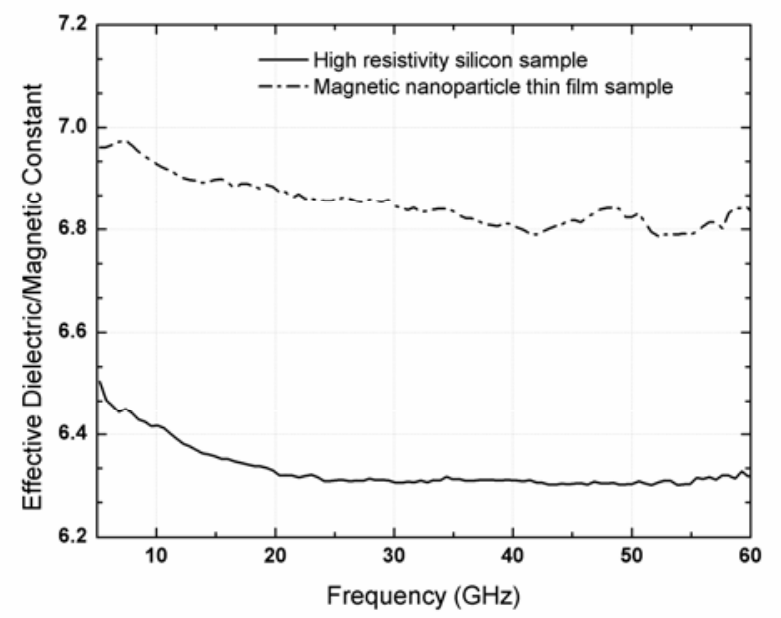

Fig. 4. Measured effective dielectric/magnetic constant. Extracted from NIST MULTICAL calibration.

The measured attenuation of the finite coplanar waveguide on high resistivity silicon with the thickness of $525 \mathrm{um}$ and on the nanoparticle thin film is shown in Fig. 5. The attenuation on the thin film is less than that on high resistivity silicon when the frequency is higher than $18 \mathrm{GHz}$ since the wave field is concentrated in the nanoparticle thin film, which has a most probably lower loss. At lower frequencies, less than $18 \mathrm{GHz}$, the difference of attenuation is relatively small. The loss in the conductor, which is dependent on the geometry of the lines and the conductivity, dominates the attenuation at low frequencies and is approximately proportional to the square root of the frequency. At high frequencies, the loss in the dielectric increases and is roughly proportional to the frequency.

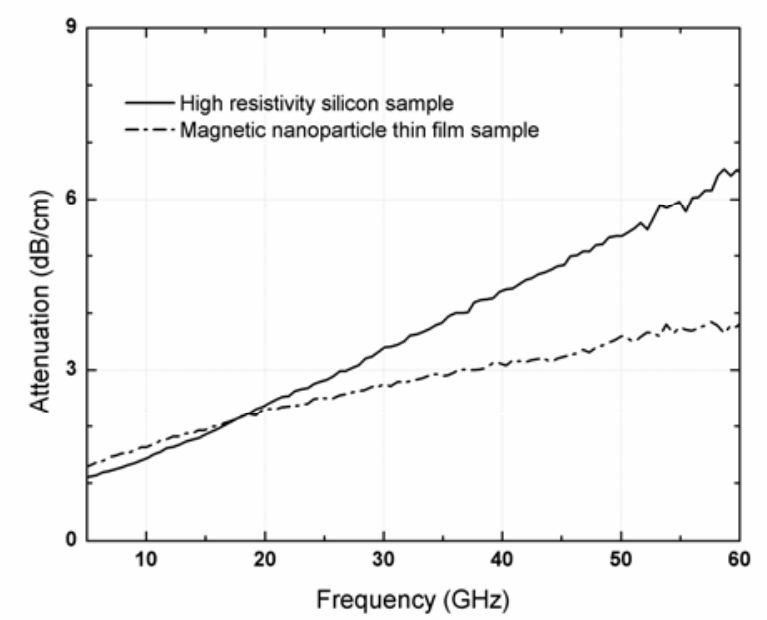

Fig. 5. Measured attenuation of the finite coplanar waveguide on high resistivity silicon and the magnetic nanoparticle thin film.

To calculate the relative permittivity of the thin film, a capacitance measurement was performed similar to the one presented in [2] and [12]. Different from [2], where Au was used as the metal layer between the thin film and the silicon, $\mathrm{Cr}$ was chosen in this paper because the thin film formed on it has better uniformity and adhesion; three metals, $\mathrm{Au}, \mathrm{Cu}$ and $\mathrm{Al}$, were also compared in our experiments. The $\mathrm{Cr}$ layer was evaporated on the silicon substrate with a thickness of $2.2 \mathrm{um}$. Once the thin film was formed on the $\mathrm{Cr}$ layer, round aluminum electrodes with diameter of $2 \mathrm{~mm}$ and thickness of $0.5 \mathrm{um}$ were evaporated with shadow mask. The capacitance between every two adjacent aluminum electrodes was measured by using a Capacitance Analyzer. To reduce the variance brought by the non-uniformity of the thin film, a large number of measurements were taken and averaged. The averaged relative permittivity of the thin film was 1.19 .

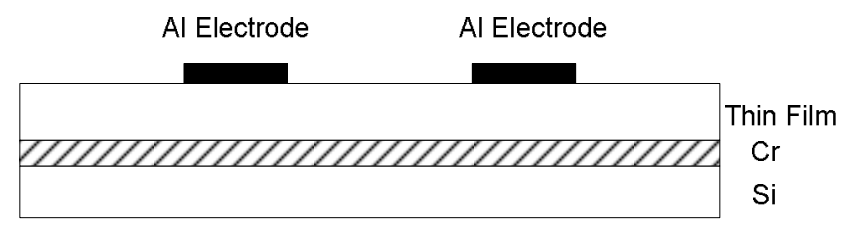

Fig. 6. Schematic of the capacitance measurement for the determination of the relative permittivity of the thin film.

\section{Simulations}

The lower loss on the thin film in comparison to that on high resistivity silicon wafer stems from the low dielectric attenuation in the thin film. The E-field distribution on the cross-section of FGC waveguide and thin film is show in Fig. 7. 

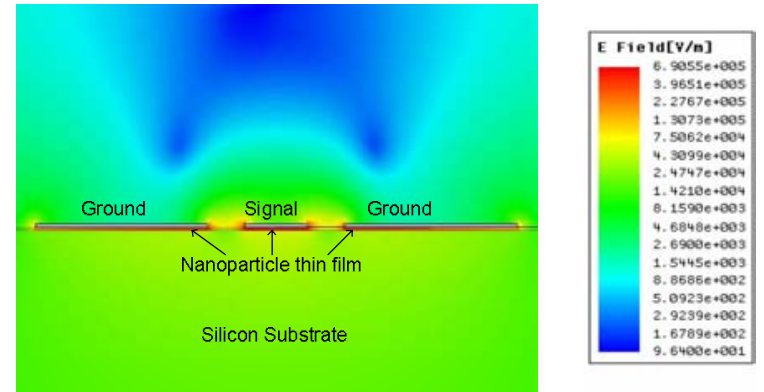

Fig. 7. Simulation of E-field on cross-section of FGC waveguide on the thin film with silicon substrate. (thickness of the thin film is exaggerated to show the concentration of the E-field in the thin film).

To determine the relative permeability of the thin film, Ansoft HFSS 10 was employed to model the finite ground coplanar waveguide with the thin film. In the simulation setup, the relative permittivity was assumed to be 1.19 and 11.9 for the thin-film and for the silicon substrate, respectively. The effective dielectric/magnetic constant of the thin film was simulated over the frequency range of $20-30 \mathrm{GHz}$. The simulated and measured dielectric/magnetic constants are shown in Fig. 8. A relative permeability with a value of 68 has matched the measured data throughout the measurement frequency range from 20 to $30 \mathrm{GHz}$. Compared to [2], the extracted relative permeability is increased by $\sim 15 \%$. The simulated characteristic impedance is increased from $51 \mathrm{ohms}$ to $55 \mathrm{ohms}$ at $30 \mathrm{GHz}$.

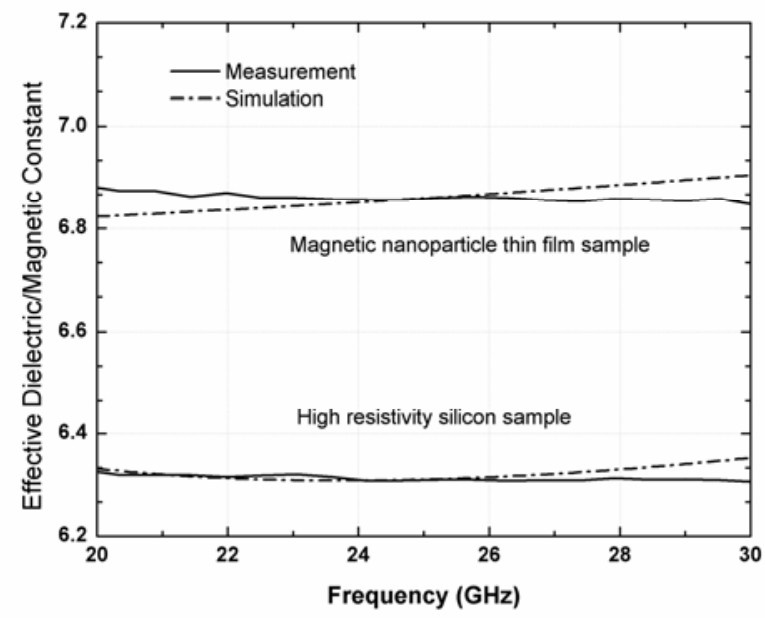

Fig. 8. Simulation and measurement of the effective dielectric/magnetic constant over the frequency range of 20-30 GHz.

\section{CONCLUSION}

This paper presents an enhanced-thickness nanoparticle based magnetic thin film for SOC type wireless applications. Experimental results have shown that for 30 layers of nanoparticle thin film, a $\mu_{r}$ of 68 and $\varepsilon_{r}$ of 1.19 were achieved. An attenuation of $0.27 \mathrm{~dB} / \mathrm{mm}$ was achieved at 30 GHz. This method can pave the way for highly compact low loss components (inductor, attenuator) on silicon substrate for wireless applications up to $60 \mathrm{GHz}$.

\section{ACKNOWLEDGEMENTS}

The authors wish to thank Dr. Matt Morton at Raytheon for the experiment, Dr. Dane Thompson at Ansoft for the simulation and Ms. Zhan Liu from Georgia Tech on the help of taking SEM pictures.

\section{REFERENCES}

[1] I. Rondinone, A.C.S. Samia, Z. J. Zhang, "Supelparamagnetic Relaxation and Magnetic Anisotropy Energy Dish-ibution in CoFe201 Spinel Ferrite Nanonystallites," J. Phys. Chem. E. vol. 103 no 33, pp. 6876-6880, 1999.

[2] M.A. Morton, C.R. Vestal, J. Papapolymerou, Z.J. Zhang "Fabrication and characterization of spinel magnetic nanoparticle thin film transmission lines," 33rd European Microwave Conference, 2003, Volume: 3, pp. 1307-1309, 7-9 Oct. 2003.

[3] M. Yamaguchi, K. Suezawa, K.I. Arai, S. Kikuchi, W.D. Li, Y. Shimada, S. Tanabe, K. Ito, J. Appl. Phys. 85 (1999) 7919.

[4] D. Gardner, A.M. Crawford, Shan Wang, Proceedings of the 2001 IEEE IITC, 2001, p. 101.

[5] I. Fergen, K. Seemann, A. v. d. Weth, A. Schuppen, Proceedings of the First Joint European Magnetic Symposia, TU-A1-I1, 2001

[6] M. Yamaguchi, K.-H. Kim, T. Kuribara, K.I. Arai, IEEE Trans. Magn. 38 (2002) 3183.

[7] K. Tan, M. Yamaguchi, K. Yamanaka, K. Ouchi, K.I. Arai, IEEE Trans. Magn. 37 (2001) 2004.

[8] M. Yamaguchi, H. Kikuchi, S. Sugimoto, K.I. Arai, M. Iwanami, A. Nakamura, S. Hoshino, Proceedings of the IEEE 10th Topical Meeting on Electrical Performance of Electronic Packaging, 2001, p. 321.

[9] Yuan Li, Pete L. Kirby, Olivier Offranc, and John Papapolymerou "Silicon Micromachined W-band Hybrid Coupler and Power Divider Using DRIE Technique," accepted and will be published at IEEE Microwave and Wireless Components Letters, Jan. 2008.

[10] R. B. Marks, "A multiline method of network analyzer calibration," IEEE Trans. Microwave Theory and Techniques, Vol. 39, pp. 1205-1215, July 1991.

[11] G. E. Ponchak, "RF transmission lines on silicon substrates," $29^{\text {th }}$ European Microwave Conference Dig., Munich, Germany, Oct. 5-7, 1999, pp. 158-161.

[12] S. Sinharoy, H. Buhay, M. G. Burke. D. R. Lamp, T. M. Poll\& "Growth and the Microstructure and Ferroelectric Characterization of Oriented BaMgF4 Thin Films," IEEE Trans. Ultrasonics, Ferroelectrics, and Frequency Control, vol. 38 no. 6, pp. 663471. Nov. 1991. 